\title{
Rol del Médico Veterinario en la inspección sanitaria de
}

\section{carnes.}

\author{
María Pía Meledandri \\ Municiplaidad de Traiguén/Pragrama PDTI INDAP, Unidad Operativa Comunal, \\ Territorio 1. Email: pia mele@hotmail.com
}

Recibido: 23 de mayo de 2017; Aceptado: 06 de junio de 2017.

El médico veterinario cumple un rol trascendental dentro de la sociedad como lo es el certificar que los alimentos (carnes, quesos, embutidos, etc.) sean aptos para consumo humano, procurando velar por la salud de los consumidores enmarcado en el área de salud pública. Con el tiempo, esta área de la medicina veterinaria ha sido relegada privilegiando áreas como la producción animal y la clínica de animales menores, por sobre la inocuidad alimentaria y prevención de zoonosis (Armstrong 2011). Cabe destacar, que es de responsabilidad primordial de los servicios veterinarios la inspección de carnes ante y post mortem de animales destinados a sacrificio (OIE 2011). Teniendo por objetivo, detectar y prevenir riegos para la salud pública, además de permitir la vigilancia de determinadas enfermedades y verificar las normas de bienestar animal (Martín y Ortiz 2011).

Un matadero, definido por Servicio Agrícola y Ganadero (2012), en su artículo 78 como: "aquellos establecimientos donde se sacrifican y faenan los animales de abasto, destinados para consumo humano" y agrega que "deberán estar habilitados de tal forma que aseguren el faenamiento y preservación higiénica de las carnes", para su normal funcionamiento, requiere de un médico veterinario acreditado por el SAG que utilice las técnicas, conocimientos y recursos de la ciencia veterinaria para proteger la salud humana, vele que los procedimientos que allí se realicen cumplan con las normas y leyes que aseguran un producto final de calidad e inocuo (ley de carnes, código sanitario de los alimentos, norma técnica 62, etc.), inspeccione los animales al ingreso a la planta (examen antemortem) y luego de ser faenados (examen postmortem), cerciorarse también, que el flujo sea continuo y que sólo sea interrumpido cuando la razón involucre un factor que pueda afectar la inocuidad del producto final. Además de encargarse de registrar todas las actividades y procesos que ocurren en la planta, para que las acciones de ésta estén respaldadas. Al certificar que las canales y 
subproductos son aptos para el consumo humano, de calidad e inocuos se evitan epidemias y enfermedades de tipo zoonóticas en la población, aumentando la esperanza de vida del ser humano mediante la promoción de la salud, la prevención y el control de enfermedades, mejoras en la producción e higiene de los alimentos y la protección del medio ambiente (Ortega et al. 2005).

\section{REFERENCIAS}

Armstrong, W 2011, 'Salud pública veterinaria actualidad e información sobre la salud pública veterinaria, con énfasis en contenidos asociados a Chile', Asociación Chilena de Zoonosis - ACHIZ [Blog], 14 de mayo, consultado: 18 junio de 2011, http://www.saludpublicaveterinaria.cl/2011/05/asociacion-chilena-dezoonosis-achiz.ht $\mathrm{ml}$ \#more.

Martín, AM y Ortiz, A J 2011, 'Protección de la salud...seguridad alimentaria y salud ambiental', Cambios futuros en la inspección de carnes [Blog], 11 de febrero, consultado: 3 marzo de 2011, http://www.protecciondelasalud.com/2011/01/cambios -futuros-en-lainspeccion-de.html.

Organización Mundial de Sanidad Animal (OIE) 2011, 'Control de riesgos biológicos que amenazan la salud de las personas y la sanidad de los animales mediante la inspección ante mortem y post mortem de las carnes' [En línea], OIE - Código Sanitario para los Animales Terrestres Artículo 6.2.1, vol. I, Chile, consultado: 2 abril de 2011, http://www.oie.int/fileadmin/Home/esp/Health_standards/tahc/2010/es_ chapitre_1.6.2.pdf.

Ortega, C; Villamil, N; Cediel, C; Rosenfeld, D; De. Meneghi, M; De Rosa, L; Estol, G., Lleguia, A; Fonseca-Poveda, M; Torres, M; Caballero-Castillo y De Balogh, K 2005, 'Las redes SAPUVET y SPVet: un modelo de integración en materia de salud pública veterinaria entre Europa y América Latina', Revista Panamericana Salud Publica/Pan Am J Public Health, 17 (1), pp. 60-65.

Servicio Agrícola y Ganadero (SAG) s.f; Fiscalización de la Ley de la Carne, [Página web], consultado: 12 mayo de 2012, http://historico.sag.gob.cl/opendocs /asp/pagDefault.asp?boton=Doc52\&argInstanciaId=52\&argCarpetaId=535\&arg TreeNodosAbiertos=\%28535\%29\%2852\%29\&argTreeNodoActual=535\&argTree NodoSel=\#Tope. 
Sustainability, Agri, Food and Environmental Research, 5(2), 2017: 41-42 ISSN: 0719-3726 